\title{
Star Cluster Simulations Including Stellar Evolution
}

\author{
Stephen L. W. McMillan \\ Department of Physics, Drexel University, Philadelphia, PA 19104
}

\begin{abstract}
The past few years have seen dramatic improvements in the scope and realism of star cluster simulations. Accurate treatments of stellar evolution, coupled with robust descriptions of all phases of binary evolution, have been incorporated self-consistently into several dynamical codes, allowing for the first time detailed study of the interplay between stellar dynamics and stellar physics. The coupling between evolution, dynamics, and the observational appearance of the cluster is particularly strong in young systems and those containing large numbers of primordial binary systems, and important inroads have been made in these areas, particularly in $N$-body simulations. I discuss some technical aspects of the current generation of $N$-body integrators, and describe some recent results obtained using these codes.
\end{abstract}

\section{Introduction: A Revolution in Cluster Modeling}

Star clusters are remarkably clean realizations of the classical $N$-body problem. They are relatively isolated in space, consist of more or less coeval stars, and contain relatively little gas or dust. As such, they provide unique laboratories for the study of fundamental stellar dynamical processes-relaxation, mass segregation, core collapse, binary interactions, post-collapse evolution, and evaporation with and without the effects of external tidal fields. Globular clusters are perhaps the best examples of "clean" systems. They are indeed isolated, gas free, and largely separate from the rest of the Milky Way Galaxy (except for tidal effects), and stellar evolution is relatively unimportant in governing their present large-scale dynamics, although it may be critical in determining the appearance of individual cluster components.

Of course, this is an oversimplified picture. In reality, cluster evolution involves not just stellar dynamics but also stellar evolution and mass loss, binary evolution, stellar collisions and tidal interactions, not to mention timedependent external influences such as molecular clouds, bulge and disk shocks, and the effects of cluster orbital evolution. Combined, this complex mixture of competitive, tightly coupled physical processes poses great difficulties to wouldbe modelers. Open clusters and the Arches and Quintuplet systems found in the Galactic nucleus are examples of such "dirty" systems (the term reflecting somewhat the prejudices of a dynamicist). Some are sites of ongoing star formation, gas dynamics is evident in others, many are still strongly influenced by stellar evolution, and almost all have relatively short lifetimes in the Galactic tidal field. Indeed, one could argue that the surviving globulars are very un- 
usual, and they are now clean only because they have survived the competitive processes that have already destroyed 99 percent of their siblings. Thus we need to study dirty systems even to understand the clean ones observed today.

Studies of cluster dynamics came of age in the 1980s, when improved simulation techniques elucidated many of the basic evolutionary processes operating in stellar systems - core collapse, mass segregation, binary dynamics, and the largescale effects of mass loss due to stellar evolution. However, for good theoretical and practical reasons, the work concentrated on problems consisting essentially of point-mass dynamics with the inclusion of a few additional well-defined effects of interest. Limited combinations of physical processes afforded better understanding of cluster dynamics, and limitations in simulation techniquesthe approximations inherent in continuum codes and the sheer computational expense of $N$-body simulations - largely dictated the simulation agenda.

The 1990s saw the integration of many detailed physical processes into simulation codes. Mass loss in evolving cluster environments, binary dynamics in clusters, and eventually the introduction of both stellar and binary evolution into $N$-body and Monte-Carlo codes. At the same time, the advent of specialpurpose GRAPE hardware has greatly increased the numbers of stars that can be followed by direct integration. The result is that realistic star-by-star simulations of open clusters and small globular clusters are now possible, and the prospects for expansion into larger globular clusters are good. Our simulations are still limited by our knowledge of detailed physics, cluster initial conditions, gas dynamics, and orbital evolution, but the state of the art today is clearly qualitatively different from the situation even five years ago.

In this paper I outline some recent advances in cluster simulation, with particular reference to the effects of stellar and binary physics. I first describe some basic dynamics and the effect of mass loss due to stellar evolution. I then turn to the simulation techniques now in use, and specifically to their incorporation of stellar and binary evolution. Finally I list some recent studies carried out using the new generation of $N$-body codes, and discuss a few issues that remain to be resolved.

\section{Cluster Dynamics and the "Kitchen Sink"}

\subsection{Dynamical Evolution}

An isolated cluster of mass $M$ consisting of non-evolving point stars comes into dynamical equilibrium on a crossing time scale $t_{D}=\left(G M / R_{v i r}^{3}\right)^{-1 / 2}$, where $R_{v i r}$ is the cluster virial radius. It evolves thermally on a relaxation time scale $t_{R} \sim(N / 8 \log \Lambda) t_{D}$, where $N$ is the number of stars in the system and $\Lambda \sim 0.1 N$ (Spitzer 1987, Giersz \& Heggie 1996).

Heat conduction from the cluster core to the outer halo precipitates the phenomenon known as core collapse, in which the cluster's central core shrinks to (formally) infinite density while the outer regions expand (Antonov 1962). The core-collapse time scale, for a cluster consisting of equal-mass stars, is $t_{c c} \sim$ $15 t_{R, h}$ (Cohn 1979), where $t_{R, h}$ is the relaxation time at the half-mass radius (comparable to the virial radius in most cases). For our purposes, the most important effect of core collapse is the creation of a dense central core, leading to enhanced stellar and binary interactions. 
Energy equipartition (dynamical friction) causes massive stars to segregate to the cluster center (Spitzer 1969). For a star of mass $m$ in a cluster of mean mass $\langle m\rangle \ll m$, the segregation time scale is $t_{\text {seg }} \sim t_{R}\langle m\rangle / m$. With even a modest range in masses, mass segregation can speed up core collapse by a factor of 3-5 or more relative to the equal-mass figure (Inagaki 1985). In systems with realistic mass spectra and broad ranges in mass ( 0.1 to 100 solar masses is typical in very young star clusters), significant mass segregation can occur in as little as $0.2 t_{R, h}$ (Portegies Zwart \& McMillan 2002a). Since mass segregation leads to the creation of a dense central core of high-mass stars, with the attendant processes of binary formation and stellar interactions, mass segregation effectively is core collapse in these cases.

Core collapse continues until some mechanism can replace the energy lost by conduction from core to halo. Leading contenders include mass loss due to stellar evolution and collisions, which tend to unbind the cluster, and heating due to binary interactions. The dynamical effect of binaries is most simply expressed by "Heggie's Law" (Heggie 1975) - the well-known statement that hard binaries (those having energies greater than the mean stellar kinetic energy, $\frac{3}{2} k T$ ) tend to become harder following interactions with other stars, while soft binaries tend to be destroyed. A population of primordial binaries can support the core at a few percent of the half-mass radius until the binaries are depleted by interactions or escape (Goodman \& Hut 1989; McMillan \& Hut 1994). Dynamical binary formation requires core collapse (or collapse of a massive subsystem) down to a few tens of stars. In either case, binaries heat the system at a roughly constant rate until they are destroyed by a collision or ejected from the system.

Cluster dynamical evolution is also strongly influenced by external (tidal) fields, which tend to strip the least bound, and often lowest mass, stars. Ultimately, tidal mass loss reverses core collapse by removing the confining cluster potential, leading to the possibility of a remnant core rich in binaries and/or massive stars if the cluster lifetime is sufficiently short (McMillan \& Hut 1984; Portegies Zwart et al. 2002a). Typically, in a stationary tidal field corresponding to a cluster moving in a circular orbit around the Galactic center, the cluster mass goes roughly linearly to zero on a time scale of a few times the "tidal relaxation time" (essentially the above expression for $t_{R}$, but using the dynamical time at the tidal radius), the details depending on the cluster's initial internal structure (Portegies Zwart et al. 2001a). Studies of time-dependent fields suggest that the effects of external tidal shocks may well be comparable to internal relaxation in determining clusțer evolution (Gnedin \& Ostriker 1997).

\subsection{Stellar Evolution}

Much useful information can be obtained by studying specific effects in isolation and, given the choice, most workers would probably prefer such an incremental approach. However, this is not possible, as there is little middle ground between models consisting of identical, non-evolving point masses and the so-called "kitchen sink" simulations in which all relevant physical effects are modeled in detail. $^{1}$

\footnotetext{
${ }^{1}$ Paradoxically, the term refers to an expression indicating completeness in which everything but the kitchen sink is included.
} 
The reason for this is not difficult to grasp. A cluster model consisting of identical point masses is not an accurate description of any real star cluster. Such a simple system has only one relevant parameter - the total number of stars, $N$. However, increasing the realism of the model by adding a spectrum of stellar masses necessarily introduces specific stellar physics into the calculationa stellar mass function must be chosen, and the spatial distribution of each stellar species defined. Once this is done, it immediately becomes necessary also to include the effects of stellar evolution-stellar evolutionary time scales are often comparable to the mass segregation/core collapse times of the parent cluster, so the dynamical state of the cluster is tightly coupled to the state of the component stars. Alternatively, neglect of stellar evolution will lead to a core populated with unphysically massive stars, whose dynamics is unrepresentative of any real system. In addition, real star clusters contain large numbers of binary systems (e.g. Rubenstein 1997) and stellar evolution drives binary evolution, so this too must be incorporated if the simulation is to remain self-consistent. Binary evolution may lead to stellar mergers and the production of a veritable zoo of exotic objects (see Portegies Zwart et al. 2001b).

Thus, the seemingly innocuous improvement of including stellar masses actually leads to a complex mix of physical processes and an exceedingly difficult numerical problem. As tidal effects and stellar physics are included, it is unclear how well our insight extends from simple to complex systems. The interplay of physical processes demands a more comprehensive approach.

\subsection{Dynamical Effects of Stellar Evolution}

The importance of stellar evolution to cluster dynamics was made clear by Applegate (1986) in a semi-analytic study of stellar mass loss from an idealized dynamical system. The study revealed that, for sufficiently flat initial mass functions (i.e. enough mass in massive stars), the combination of máss segregation and mass loss was sufficient to unbind a cluster long before core collapse could occur. For stellar masses distributed as a power law, $d N / d m \sim m^{-\alpha}$ for $0.35 M_{\odot} \lesssim m \lesssim 4 M_{\odot}$, Applegate found that the dividing line between core collapse and disruption lay at $\alpha \sim 2$ (where the slope of the Salpeter mass function is $\alpha=2.35$ ).

These conclusions were refined and strengthened by Chernoff \& Weinberg (1990), who carried out the first systematic survey of globular cluster survival in Galactic environment with the effects of stellar evolution included selfconsistently into a dynamical model. They employed a Fokker-Planck approximation with a simplified "main-sequence to giant to remnant" description of stellar evolution and an approximate "absorbing-boundary" treatment of the Galactic tidal field. Their model clusters concentrated on initially unsegregated King (1966) models with dimensionless depths $W_{0}=1,3$, or 7 (corresponding to a range in cluster Galactocentric radii of $2-25 \mathrm{kpc}$ for the cluster masses considered), with initial stellar masses in the range $0.4 M_{\odot} \leq m \leq 15 M_{\odot}$, distributed as power-laws with $\alpha=1.5,2.5$, or 3.5. They found that all clusters with $W_{0}=1$ or $\alpha=1.5$ dissolved before core collapse, while all clusters with $W_{0}=7$ and $\alpha>1.5$ collapsed. For $W_{0}=3$, the dividing line between disruption and survival for a Hubble time lay at $\alpha$ somewhat greater than 2.5, and those clusters that did survive lost most of their mass by the present day. 
The first systematic series of $N$-body calculations incorporating stellar evolution was carried out by Terlevich (1987) using Aarseth's NBODY5 integrator (Aarseth 1985). A variety of power-law initial mass functions was considered, and some models were started out of dynamical equilibrium (an example of an initial condition inaccessible to most other modeling techniques). Computational limitations restricted the systems studied to $\lesssim 1000$ stars, but the models clearly demonstrated the same processes of mass segregation, mass loss, and cluster dissolution seen in Applegate's semi-analytic calculation and Chernoff \& Weinberg's Fokker-Planck simulations. Model lifetimes were in good general agreement with observed open clusters, and early termination of core collapse due to stellar evolution was evident.

As available computing power increased, it became possible to compare Fokker-Planck simulations directly with $N$-body calculations. The $N$-body studies by Fukushige and Heggie (1995) and Portegies Zwart et al. (1998) included more sophisticated treatments of stellar evolution and external tides, although they were still limited to the low-mass end of Chernoff \& Weinberg's study. Fukushige and Heggie chose initial model parameters (mass range, $\alpha$, and $W_{0}$ ) largely identical to those of Chernoff \& Weinberg; the models of Portegies Zwart et al. used similar (King model) cluster initial profiles, but adopted a more realistic initial mass function and a more sophisticated treatment of stellar evolution. Overall, both studies found general qualitative agreement with the conclusions of Chernoff \& Weinberg, although they disagreed in detail, particularly in cases where cluster disruption occurred rapidly (and the FokkerPlanck approximation is least applicable), when the $N$-body lifetimes exceeded the Fokker-Planck estimates by as much as an order of magnitude.

De la Fuente Marcos (1997) performed studies of the evolution of small, tidally limited open clusters having a variety of initial mass functions, with and without the inclusion of stellar (but not binary) evolution. All models started with a substantial fraction $(1 / 3)$ of primordial binaries having energies in the $\sim 1-10 k T$ range. He found that the dissolution time scale of his models depended quite sensitively on the choice of IMF, and that the binary population shortly before dissolution could show characteristic features allowing remnants of rich and poor clusters to be distinguished observationally. It is unclear how these results extend to larger systems.

\section{Realistic Cluster Simulations}

Given that stellar mass loss is of crucial importance to cluster evolution, and the basic ingredients have been known for over a decade, one might reasonably wonder why it has taken so long for stellar and binary evolution to be fully integrated into dynamical simulations. The short answer is that the methods best equipped to handling the physics have until been recently been unable to follow sufficiently large numbers of stars, as I now discuss.

\subsection{Simulation Methods}

The author's biases notwithstanding, a fairly convincing case can be made that $N$-body codes are particularly well suited to the inclusion of arbitrarily complex stellar (and other) physics. They make no simplifying assumptions about the 
geometry or physical state of the system under study, include all interactions to all orders, and can in principle be extended to incorporate any desired process. The price of this is computational expense. At least for open clusters, and possibly also for larger systems, it appears that direct $N^{2}$ force methods are necessary for accurate long-term (post-collapse) collisional calculations.

Tree codes (e.g. Barnes \& Hut 1986) are a possible alternative with superior $[O(N \log N)]$ scaling, but they introduce spatial and temporal correlations in interparticle forces, possibly causing spurious diffusion effects which may be important during the post-collapse phase. McMillan \& Aarseth (1993) found that a tree code could successfully reproduce the main features of cluster core collapse, and specifically the collapse time scale, but their study did not probe the post-collapse regime. However, they found that trees are inherently poorly suited to systems with individual particle time steps and large dynamic ranges in space and time scales, leading to significant complexity in the implementation of their tree code. This latter consideration also applies to fast multipole methods (Greengard \& Rokhlin 1987), which generally perform best in homogeneous systems having limited dynamic ranges.

Direct-summation codes received an enormous boost in the 1990s with the development of the GRAPE series of special-purpose computers (Makino et al. 1997), the reason for this conference and the subject of numerous contributions in these proceedings. Abandoning algorithmic sophistication in favor of raw parallel computing power, the GRAPE family of processors increase the size of $N$-body systems by perhaps an order of magnitude over what is feasible using conventional supercomputer technology. The advent of GRAPE has greatly alleviated the computational bottleneck in $N$-body simulations, making it possible to focus on the physics rather than the numerics, and today GRAPEs are at the heart of all detailed simulations of star clusters and dense stellar systems. The current state of the art in collisional $N$-body simulations is $N \sim 10^{5}$.

Two popular competitors to direct summation are the use of direct FokkerPlanck codes and gas-sphere methods. In the former, the effects of repeated stellar encounters are distilled into a set of phase-space diffusion coefficients and the evolution of the system is followed using the Fokker-Planck equation in phase space (Cohn 1979). The latter methods transform the collisional Boltzmann equation into a system of moment equations closely resembling those of stellar structure, with stellar interactions providing the means for heat to flow around the system (Bettwieser \& Sugimoto 1984). Both approaches are much cheaper than $N$-body techniques. However, at least in their simplest forms, they are not well suited to full treatments of cluster evolution. They rapidly become complex, unwieldy, and of questionable statistical validity as new dimensions are introduced to accommodate stellar masses, binary interactions, stellar collisions, etc. In addition, they admit only approximate treatments of tidal fields and tidal stripping, although Takahashi and Portegies Zwart (1998) have demonstrated that the tidal mass-loss rates can be largely reconciled with detailed $N$-body results if proper care is exercised in applying the stripping criterion.

Monte-Carlo methods provide, depending on one's point of view, an alternative means of integrating the Fokker-Planck equation or a more faithful realization of the probability distributions underlying it. Either way, they represent promising "middle ground" between $N$-body and continuum schemes, combin- 
ing the ability to model large systems with treatments of many physical effects. Current codes include both "pure" Monte-Carlo methods (Joshi, Nave, \& Rasio 2001, Giersz 2001) and "hybrid" approaches combining Monte-Carlo treatments of selected stellar species (binaries, compact remnants, etc.) with continuous descriptions of the rest of the system (Giersz \& Spurzem 2000). As yet these schemes do not include detailed treatments of stellar and binary evolution, but those developments are anticipated within the next few years.

Thus-for now, at least- $N$-body methods seem to offer the best means of including detailed stellar physics in cluster simulations, despite their continuing limitations in system size. Larger simulations using Monte-Carlo methods are possible, and may include more detailed physical modeling soon, but such methods are still not well suited to systems characterized by large departures from spherical symmetry or dynamical equilibrium.

\subsection{N-body Modeling}

The Aarseth "NBODY" series of $N$-body integrators (NBODY1-6) and their offshoots (NBODY6++ and others) are widely known and quite thoroughly documented (see Aarseth 1999 and Hurley, these proceedings). I will concentrate here on describing the "Starlab" software suite (Portegies Zwart et al. 2001b) and some of its similarities and differences from the Aarseth codes. While written as an independent alternative to the NBODY series, Starlab shares many structural and algorithmic features with it-perhaps not surprising, since Sverre Aarseth has over the past three decades developed and refined many of the key elements of all $\mathrm{N}$-body simulations. However, some significant differences exist too.

Starlab is a collection of modular software tools designed to simulate the evolution of stars and stellar systems and to analyze the resulting data. The package consists of a library of loosely coupled programs, sharing a common flexible data structure, which can be combined in arbitrarily complex ways to study the dynamics of binary and multiple star systems, star clusters and galactic nuclei. Central to Starlab is the kira integrator, whose key features include

- a fourth-order Hermite integrator (Makino \& Aarseth 1992) using a block time step scheduling scheme (McMillan 1986)

- a tree-based data structure, with single stars and binary centers of mass forming the top-level nodes in the tree, replacing the simple arrays used in the NBODY codes

- use of GRAPE hardware for top-level nodes (when available)

- computation of all low-level (binary) motion relative to the center of mass

- homogeneous treatment of binaries and multiples of arbitrary complexity using a binary tree structure; the use of relative coordinates and unperturbed motion where appropriate obviates the need for the binary, triple and chain regularization schemes employed in the NBODY codes

- use of a self-consistent hierarchical unperturbed approximation for efficient treatment of isolated binaries and multiples 
- an efficient treatment of lightly perturbed binaries, modeled on the "slow KS" formalism of Mikkola \& Aarseth (1996)

- stellar tidal interactions and collisions

- comprehensive treatments of stellar and binary evolution

The package is described in considerably more detail, and is available for download, at http://manybody.org.

As in NBODY4, stellar evolution is incorporated into kira via look-up from precomputed tracks, interpolating in age and mass as described by Eggleton, Fitchett, \& Tout (1989), and in metallicity following Hurley, Pols, \& Tout (2000; see also Hurley, these proceedings). Stars and binaries are evolved in time at regular intervals or as needed, depending on individual circumstances (e.g. following a merger in a binary or a collision of unbound stars).

Binary evolution in kira follows the work of Portegies Zwart (1997), and includes internally consistent treatments of all relevant evolutionary phases. Detached binaries may experience tidal circularization (Zahn 1978), slow mass loss due to stellar winds, more rapid mass loss as a star becomes a white dwarf, or instantaneous mass loss in a supernova explosion (Hills 1983). The velocity "kick" that neutron stars, and possibly also black holes, receive at birth is taken from Hartman (1997). Angular momentum loss may occur through stellar winds, magnetic braking (Rappaport, Verbunt, \& Joss 1983), or gravitational radiation (Peters \& Mathews 1963). For semi-detached binaries, we include the possibility of tidal instability and a comprehensive treatment of stable and unstable mass transfer. Accretion disk formation around compact companions is also modeled. The structure of the package is such that evolutionary recipes can be updated and refined as theoretical insights improve, without necessitating major modifications to the rest of the program.

Cross-sections for, and the most probable outcomes of, stellar collisions not resulting from binary evolution are taken wherever possible from published simulations, mainly using smoothed-particle hydrodynamics (e.g. Lombardi, Rasio, \& Shapiro 1996, Freitag \& Benz 2000, Sills et al. 2001). Results in the literature tend to concentrate on collisions of main-sequence stars and selected encounters involving normal stars and specific compact objects of interest $\left(0.5 M_{\odot}\right.$ white dwarfs, $1.4 M_{\odot}$ neutron stars, etc.). For other collisions, we attempt to interpolate between published results or choose the "closest" published calculation, or we simply assign effective radii to the stars involved (so approach within the sum of the effective radii implies merger).

These approximations are far from perfect, especially when one recognizes that encounters in simulations usually occur in bound systems, sometimes involving multiple stars, and thus rarely have orbital parameters similar to those adopted in published results (which typically assume parabolic orbits). One can envisage more sophisticated treatments, in which SPH or other simulations are performed on the fly as needed and the resulting object is followed back to thermal equilibrium using a stellar evolution code after re-integration into the $N$-body system. However, there are numerous technical difficulties with this approach, not the least of them being the problems of how to recognize automatically that an encounter is "over," and how to characterize and evolve the merger product. 
These issues are particularly critical in studies of runaway mergers, where one massive star grows by consuming other stars in the core of a young dense cluster (Portegies Zwart et al. 1999). Here, detailed collision cross sections, merger probabilities, the thermal evolution of the merger product, and the evolution of very massive stars may all play important roles in determining the final outcome. For now, we must simply try various parametrizations to assess the robustness of the result, but it is hoped that these approximations can be improved in future work.

Finally, we adopt a more or less agnostic attitude toward tidal capture (Fabian, Pringle, \& Rees 1975; Press \& Teukolsky 1977), the process of binary formation via tidal dissipation in an unbound close encounter, whose effectiveness as a means of forming close binaries remains controversial. Potential applications include the formation of LMXBs and CVs in the denser globular clusters (Verbunt \& Hut 1987). However, the process requires orbital separations comparable to the radii of the stars involved, so the possibility of collisions between the (probably thermally expanded) stars places a lower limit on the allowed separation (McMillan, McDermott, \& Taam 1987; Ray, Kembhavi, \& Antia 1987). An upper limit is imposed by the possibility that the binary becomes unbound on subsequent periastron passages (Kochanek 1992). The circularization process is discussed in detail by Mardling (1995) and Mardling \& Aarseth (2001), and their results are incorporated into NBODY4. In kira, the unknown stellar state following the first encounter is viewed as the primary factor limiting our knowledge of the process, and we generally simply increase the effective stellar collision radius, although we retain the option (usually switched off) of a more detailed treatment should models improve.

\subsection{Some Applications}

Rather than describing a few specific applications of the modeling techniques just described, I simply list here some recent examples and refer the reader to more detailed accounts elsewhere in these proceedings and in the literature.

Studies of open clusters range from simulations of the dynamics and appearance of small systems such as the Pleiades, Hyades, and Praesepe (Portegies Zwart et al. 2001b, 2002b; Starlab) to a detailed analysis of binaries and blue stragglers in M67 (Hurley, these proceedings; Hurley et al. 2001; NBODY4). Shara \& Hurley (2002; see also Shara, these proceedings; NBODY4) have found that dynamical interactions in clusters containing primordial binaries can lead to a substantial population of white-dwarf binaries - greatly exceeding expectations based on binary evolution alone - with inspiral time scales less than a Hubble time. If these results can be scaled to globular clusters, they may imply an interesting new channel for the production of Type Ia supernovae.

Portegies Zwart et al (1999; Starlab) performed simulations of young clusters, modeling the evolution of R136 in the 30 Doradus region of the LMC. They found that, for sufficiently dense (but not unreasonably so) initial conditions, massive stars can segregate to the cluster center and undergo runaway merger, leading to the formation of stars having masses hundreds of times that of the Sun in less then a few million years. More recently, Johnson et al. (2001) have compared population-synthesis and dynamical simulations of the young star clusters NGC 1805 and 1818 in the LMC with HST observations of these systems. 
Portegies Zwart et al. (2001a, 2002a; see also Portegies Zwart, these proceedings; Starlab) have performed a series of simulations of the lifetimes and visibility of Arches- and Quintuplet-like clusters near the Galactic center, concluding that the central regions of our Galaxy may harbor dozens of similar clusters below the threshold of visibility. Subsequent studies by Portegies Zwart \& McMillan (2002) and McMillan \& Portegies Zwart (2002) have followed both the inspiral of such clusters into the Galactic Center and the possible formation of intermediate-mass black holes following runaway mergers in their cores. Ebisuzaki et al. (2002; see also Ebisuzaki, these proceedings) have extended these ideas to the formation and subsequent inspiral of intermediate-mass black holes in M82 and other galaxies.

\section{The Future}

The state of the art in cluster simulations has made impressive strides, but many outstanding issues remain. I conclude by listing, in no particular order, some significant challenges to be addressed in the next few years.

\subsection{Cluster Initial Conditions}

All simulations necessarily make simplifying assumptions about the initial state of the cluster under study. They start with a specific choice of IMF (powerlaw, Scalo, Kroupa, etc.) usually based on observations of stars in the solar neighborhood. All stars are assumed to lie on the zero-age main sequence, the cluster is taken to be gas-free and in dynamical equilibrium (but see Kroupa, Aarseth, \& Hurley 2001), the density profile takes on some simple standard form (e.g. Plummer, King, anisotropic King) and generally is applied to stars of all masses. Binary parameters are chosen from convenient (but poorly known) distributions, with binary components typically drawn randomly from the IMF and binary spatial densities following the master density profile of cluster stars. Of course, most of these assumptions are incorrect.

- There is no reason to suppose that the local stellar mass function or binary population is representative of initial mass functions in young globular clusters (billions of years ago), or in dense massive systems forming today (see Kroupa 2001 for a thorough discussion).

- Stars of different masses do not all reach the zero-age main sequence simultaneously, so the births of the "coeval" stars in our models may easily span 100 Myr or more, longer than the cluster lifetime in some cases; protostellar disks may have large cross sections and might contribute significantly to collisional mergers.

- Many, if not most, young star clusters are rich in gas whose eventual expulsion will cause the cluster to expand, and perhaps dissolve. Thus, clusters are probably not in dynamical equilibrium at birth (or when stellar dynamics is typically "turned on"), and gas dynamics should be routinely incorporated into models of early cluster evolution.

- It is also possible that clusters are born mass segregated, with more massive stars forming in the densest regions of the parent cloud (see the discussions 
by Klessen and Whitworth, these proceedings), so the assumption of a universal density profile may not be warranted. In that case, the use of mass segregation as an indicator of dynamical evolution must also be reconsidered.

\subsection{Better Treatments of Stellar Physics}

As described above, look-up tables, semi-analytical recipes, and specific simulations now form the basis for the inclusion of stellar and binary evolution and collisions into cluster simulations. Can we do better? Might actual stellar evolution, SPH, even binary evolution codes be embedded in $N$-body and other integrators? Technically, such an interface would be quite feasible -for example, the internal structure of kira was designed with the inclusion of both stellar evolution and SPH in mind - although a clear formulation of the possible interactions between the various modules involved is critical.

The basic challenge of such an approach is to build a robust standalone module that can function reliably and reasonably no matter how unexpected or unreasonable the input might be. While numerous capable stellar evolution packages exist, none is intended to follow the evolution of an object from birth to death without user intervention at certain key stages. Nevertheless, such a self-contained program could probably be constructed, given a sufficiently motivated programmer. Experiments with Starlab indicate that SPH can also be incorporated, although this possibility has never been pursued to a final working product. Binary evolution appears much harder, as the physics is less well understood, and the prospect of robust automatic evolution seems remote.

If any of this turns out to be feasible, we should then ask if it is desirable. The obvious advantage is that we can follow in detail the evolution of any object, be it an original star or the result of a later merger, without the need for look-up tables or approximate "rejuvenation" schemes for locating a merger product in an existing array of precomputed models. On the other hand, the automatic module, in part by the very nature of the robustness needed for standalone use, may result in models of lower resolution than those underlying the lookup tables. In addition, one could argue that the ability to parametrize physical processes into recipes allows us the freedom to experiment with individual effects (much as Monte-Carlo methods do with dynamics) that perhaps could not be isolated within a more complete evolution treatment. Most probably the coming $2-3$ years will see the development of hybrid methods combining look-up and automated computation of stellar structure as needed.

\subsection{Scaling and Calibration}

Even with GRAPE-6, it is still not feasible to model large globular clusters using $N$-body methods. Can we scale up smaller runs to tell us about larger systems? In the early 1990s, the hope was that studies of cluster evolution of steadily increasing size would lead to the development of simple scaling relations that would permit extrapolation of $N$-body results to larger values of $N$. Unfortunately, it now seems that there are too many "important" time scales for that to be possible: in addition to the dynamical and relaxation time scales ( $\$ 2.1)$, cluster evolution also depends on the evolutionary time scales $t_{S}$ for individual stars, the time scales for collisions between stars, and the time scale on which 
stars are stripped by an external field. All of these scales depend on the choice of stellar mass function and other cluster parameters.

The ratio $t_{R} / t_{D}$ is set by the value of $N$. The basic approach used by researchers since Chernoff \& Weinberg (1990) has been to try to preserve one of the ratios $t_{S} / t_{D}$ or $t_{S} / t_{R}$. However, Portegies Zwart et al. (1998) found that the scaling even from $4 \mathrm{k}$ to $16 \mathrm{k}$ is unreliable in presence of tides and stellar mass loss, whichever of these two ratios is held fixed. Variable scaling (Aarseth \& Heggie 1998), in which one adjusts the conversion between stellar and dynamical time scales as the evolution proceeds and the system makes a transition from "dynamical" to "relaxation" scaling, may work in certain controlled situations, but it appears inadequate in the presence of primordial binaries, binary evolution, and the possibility of stellar collisions. The introduction of time-dependent external fields only makes matters worse. With scaling efforts currently in doubt, there seems no alternative to doing it the hard way, in which case realistic $N$-body models of million-body systems may have to await the arrival of the GRAPE-8.

Other methods, especially Monte-Carlo codes, have already reached the million-body mark (see Giersz 2001), and we can expect them to incorporate realistic stellar physics in due course, as already discussed. However, in light of the simplifying assumptions made by these methods, $N$-body calibration will be critical as the scope of the effort expands. With more than one independent version of each major type of code now available, direct comparison of different simulation methods (e.g. Heggie, these proceedings) becomes both feasible and essential.

\subsection{Modeling Individual Systems}

Finally, it is likely that the coming years will see increasing numbers of end-toend simulations of specific systems, with the goal of making direct comparison with observations. Examples of such studies can be found in the recent simulations reported by Hurley et al. (2001), Portegies Zwart et al. (1999, 2001ab), and Johnson et al. (2001). While $N$-body studies of the larger globular clusters remain some years away, a number of intermediate-scale scale studies of systems containing $\sim 1-2 \times 10^{5}$ stars are now in progress, with stellar physics and realistic (i.e. non-circular) cluster orbits taken properly into account. As the realism and resolution of simulations increase, visualization techniques such as those described by by Levy (these proceedings) will grow in importance, as will the use of standardized data-archiving tools to analyze and distribute simulation results (Teuben, these proceedings).

Acknowledgments. This work was supported in part by grants NAG59264 and NAG5-10775 from the NASA Astrophysics Theory Program, and by the Sloan Foundation. I also gratefully acknowledge the hospitality of Tokyo University and the American Museum of Natural History in New York.

\section{References}

Aarseth, S. J. 1985, in Multiple Time Scales, eds. J. U. Brackbill \& B. I. Cohen (New York: Academic)

Aarseth, S. J. 1999, PASP, 765, 1333 
Aarseth, S. J. \& Heggie, D. C. 1998, MNRAS, 297, 794

Antonov, V. A. 1962, Vestn. Leningr. Gros. Univ., 7, 135

Applegate, J. H. 1986, ApJ, 301, 132

Barnes, J., \& Hut, P. 1986, Nature, 324, 446

Bettwieser, E., \& Sugimoto, D. 1984, MNRAS, 208, 493

Chernoff, D. F., \& Weinberg, M. D. 1989, ApJ, 351, 121

Cohn, H. N. 1979, ApJ, 234, 1036

De la Fuente Marcos, R. 1997, A\&A, 322, 764

Ebisuzaki, T., Makino, J., Tsuru, T. G., Funato, Y., Portegies Zwart, S., Hut, P., McMillan, S., Matsushita, S., Matsumoto, H., \& Kawabe, R. 2002, ApJ, 562, L19

Eggleton, P. P., Fitchet, M. J., \& Tout, C. A. 1989, ApJ, 347, 998

Fabian, A. C., Pringle, J. E., \& Rees, M. J. 1975, MNRAS, 192, 15p

Freitag, M., \& Benz, W. 2000, in Dynamics of Star Clusters and the Milky Way (STAR2000), ed. S. Dieters, B. Fuchs, A. Just, R. Spurzem, \& R. Wielsn (San Francisco: ASP), p. 428

Fukushige, T., \& Heggie, D. C. 1995, MNRAS, 276, 206

Giersz, M., \& Heggie, D. C. 1996, MNRAS, 279, 1037

Giersz, M., \& Spurzem, R. 2000, MNRAS, 317, 581

Giersz, M. 2001, in STAR2000, p. 61

Gnedin, N. Y., \& Ostriker, J. P. 1997, ApJ, 474, 223

Goodman, J., \& Hut, P. 1989, Nature, 339, 40

Greengard, L. \& Rokhlin, V. 1987, J. Comp. Phys., 73, 325

Hartman, J. W. 1997, A\&A, 322, 127

Heggie, D. C. 1975 , MNRAS, 173, 729

Hills, J. G. 1983, ApJ, 267, 322

Hurley, J. R., Pols, O. R., \& Tout, C. A. 2000, MNRAS, 315, 543

Hurley, J. R., Tout, C. A., Aarseth, S. J., \& Pols, O. R. 2001, MNRAS, 323, 630

Inagaki S. 1985, in IAU Symposium 113, Dynamics of Star Clusters, eds. J. Goodman and P. Hut (Dordrecht: Reidel)

Johnson, R. A., Beaulieu, S. F., Gilmore, G. F., Hurley, J., Santiago, B. X., Tanvir, N. R., \& Elson, R. A. W. 2001, MNRAS, 324, 367

Joshi, K. J., Nave, C. P., \& Rasio, F. A. 2001, ApJ, 550, 691

King, I. R. 1966, AJ, 71, 64

Kochanek, C. S. 1992, ApJ, 385, 604

Kroupa, P. 2001, MNRAS, 322, 231

Kroupa, P., Aarseth, S. J., \& Hurley, J. R. 2001, MNRAS, 321, 699

Lombardi, J. C., Jr., Rasio, F. A., \& Shapiro, S. L. 1996, ApJ, 468, 797

Makino, J., \& Aarseth, S. J. 1992, PASJ, 44, 141

Makino, J., Taiji, M., Ebisuzaki, T., \& Sugimoto, D. 1997, ApJ, 480, 432

Mardling, R. A. 1995, ApJ, 450, 722

Mardling, R. A., \& Aarseth, S. J. 2001, MNRAS, 321, 398 
McMillan, S. L. W. 1986, ApJ, 306, 552

McMillan, S. L. W., McDermott, P. N., \& Taam, R. E. 1987, ApJ, 318, 261

McMillan, S. L. W., \& Aarseth, S. J. 1993, ApJ, 414, 200

McMillan, S. L. W., \& Hut, P. 1994, ApJ, 427, 793

McMillan, S. L. W., \& Portegies Zwart, S. F. 2002, in preparation

Mikkola, S., \& Aarseth, S. J. 1996, Celest. Mech., 64, 197

Peters, P. C., \& Mathews, J. 1963, Phys. Rev. D, 131, 345

Portegies Zwart, S. F. 1997, Ph.D. Thesis, University of Amsterdam

Portegies Zwart, S. F., Hut, P., McMillan, S. L. W., \& Verbunt, F. 1997, A\&A, 328,143

Portegies Zwart, S. F., Hut, P., Makino, J., \& McMillan, S. L. W. 1998, A\&A, 337,363

Portegies Zwart, S. F., Makino, J., McMillan, S. L. W., \& Hut, P. 1999, A\&A, 348,117

Portegies Zwart, S. F., Makino, J., McMillan, S. L. W., \& Hut, P. 2001a, ApJ, 546, L101

Portegies Zwart, S. F., McMillan, S. L. W., Makino, J., \& Hut, P. 2001b, MNRAS, 321, 199

Portegies Zwart, S. F., Makino, J., McMillan, S. L. W., \& Hut, P. 2002a, ApJ, 565,265

Portegies Zwart, S. F., McMillan, S. L. W., Makino, J., \& Hut, P. 2002b, in preparation

Portegies Zwart, S. F. \& McMillan, S. L. W. 2002, astro-ph/0201055

Press, W. H., \& Teukolsky, S. A. 1977, ApJ, 213, 183

Rappaport, S., Verbunt, F., \& Joss, P. 1983, ApJ, 275, 713

Ray, A., Kembhavi, A., \& Antia, H. 1987, A\&A, 184, 164

Rubenstein, E. P. 1997, PASP, 109, 933

Shara, M., \& Hurley, J. 2002, astro-ph/0202179

Sills, A., Faber, J. A., Lombardi, J. C. Jr., Rasio, F. A., \& Warren, A. R. 2001, ApJ, 548, 323

Spitzer, L., Jr. 1969, ApJ, 158, L139

Spitzer, L., Jr. 1987, Dynamical Evolution of Globular Clusters, Princeton University Press

Takahashi, K., \& Portegies Zwart, S. 1998, ApJ, 503, L49

Terlevich, E. 1987, MNRAS, 224, 193

Verbunt, F., \& Hut, P. 1987, in IAU Symposium 125, The Origin and Evolution of Neutron Stars, eds. D. J. Helfand \& J. H. Huang (Dordrecht: Reidel)

Zahn, J. P. 1978, A\&A, 67, 162 